\title{
The anatomical compartments and their connections as demonstrated by ectopic air
}

\author{
Ana Frias Vilaça • Alcinda M. Reis • Isabel M. Vidal
}

Received: 4 April 2013 / Revised: 10 July 2013 / Accepted: 17 July 2013 / Published online: 25 September 2013

(C) The Author(s) 2013. This article is published with open access at Springerlink.com

\begin{abstract}
Air/gas outside the aero-digestive tract is abnormal; depending on its location, it is usually called emphysema, referring to trapped air/gas in tissues, or ectopic air/ gas. It can be associated to a wide range of disorders, and although it usually is an innocuous condition, it should prompt a search for the underlying aetiology, since some of its causes impose an urgent treatment. In rare instances, it may itself represent a life-threatening condition, depending on the site involved and how quickly it evolves. Abnormal air/gas beyond viscera and serosal spaces, reaches its location following some anatomic boundaries, such as fascia, which may help search the source; however if the air pressure exceeds the strength of the tissues, or the time between the aggression and the imaging is too long, the air/gas is almost everywhere, which may hinder its cause. Good knowledge of the anatomic spaces and how they connect between them facilitates the quick detection of the cause.

Teaching points

- Ectopic air can be depicted on conventional radiographs; but CT is more sensitive and accurate

- Visceral and retropharyngeal spaces directly communicate with mediastinum

- Renal fascia is a single multilaminated structure, which contains potential space
\end{abstract}

\footnotetext{
A. Frias Vilaça $(\bowtie)$

Department of Imagiology, Hospital-Escola da Universidade

Fernando Pessoa, Gondomar, Porto, Portugal

e-mail: ana_rita_couto@hotmail.com

A. Frias Vilaça • A. M. Reis • I. M. Vidal

Department of Radiology, Centro Hospitalar de Entre Douro e Vouga, Rua Dr Cândido Pinho, 4520-211 Santa Maria da Feira, Portugal
}

Keywords Subcutaneous emphysema $\cdot$ Pneumomediastinum Pneumoretroperitoneum · Fascia $\cdot$ Anatomy

\section{Introduction}

Air/gas is normally seen in some body structures, such as in paranasal sinuses, and in the respiratory and gastrointestinal tract. However, when present in subcutaneous tissues, cervical, mediastinal, retroperitoneal, extraperitoneal abdomen and pelvis spaces, involving muscular fibres or interstitial tissues, it is abnormal, indicating a "pathological process", and represents a challenge to search for the underlying aetiology.

Based on the embryologic development, an anatomical continuum exists between the spaces allowing air/ gas to spread along them. Since air/gas may be found distant from its point of origin, a detailed anatomical knowledge is crucial in the assessment of patients with emphysema/ectopic air/gas, contributing to a clear and attempted diagnosis.

In this article, we will briefly list local sources of ectopic air/gas in each space, followed by a revision of the local anatomy, highlighting the main connections among spaces.

\section{Pathogenesis and imaging}

Even though the word emphysema does not differentiate its composition, gas is different from atmospheric air, in both source and composition. Gas consists of carbon dioxide and nitrogen, produced by fermentation of glucose by some bacteria [1].

Several clinical conditions may present with emphysema (listed on the respective anatomic space). Often the cause is 
iatrogenic, a well-known risk of certain procedures; infection and "high pressure" movements also play an important role.

Pathologically, one of the following mechanisms might be implicated: (1) disruption of cutaneous or mucosal barriers ${ }^{1}$ (e.g. bone fractures, injectable administration, drainage catheters, complications of intubation, endoscopic and surgical procedures, gastrointestinal tract tear); (2) infectious process with gas forming microorganisms; or (3) spontaneous alveolar rupture, when the pressure gradient between air-filled alveoli and their surrounding interstitial space is sufficient to cause alveolar rupture [2].

Solid parenchymatous organs and serous membranes have a relatively great resistance to the diffusion of air/gas. Instead, air diffusion is easy through the least resistant loose areolar and fascial structures and through the communicating neurovascular structures where the fascia is anatomically discontinued [3-5].

Ectopic air/emphysema can be depicted on conventional radiographs. However, computed tomography (CT) is highly sensitive in the detection of abnormal air/gas, especially in "lung window" settings (window width, 1,500 HU; window centre, $-800 \mathrm{HU}$ ). Soft-tissue windows such as mediastinal (window width, $400 \mathrm{HU}$; window centre, $40 \mathrm{HU}$ ) and abdominal (window width, $350 \mathrm{HU}$; window centre, $50 \mathrm{HU}$ ), with their better spacial resolution contributes to specific depiction of the anatomical location and extent [6] (Fig. 1).

\section{Clinical impact and treatment}

When leakage of air/gas is greater than reabsorption, progressive accumulation in various tissue planes occurs. Commonly, subcutaneous tissue offers the least resistance to expansion.

It is usually a benign and self-limited condition, causing painless swelling of the tissues and, once its cause is resolved, in the majority of patients it spontaneously resolves in a few days (Fig. 2); high-flow oxygen therapy facilitates reabsorption of nitrogen from the distended tissues.

However, if rapidly onset, it can be associated with extreme discomfort, disfigurement, anxiety and, rarely, compartmental syndrome, especially in the neck, mediastinum and chest wall, compromising the upper airway and jugular venous drainage (raising intracranial pressure), reducing the cardiac output by compressing the heart and great vessels, and restricting the ventilation, or even causing pacemaker malfunction $[5,7,8]$. In children, whose tracheal rings are less resistant, massive pneumomediastinum may lead to compression and obstruction of the trachea-bronchial tree [9]. These emergencies

\footnotetext{
${ }^{1}$ The mechanism for the appearance of air/gas in serosal cavities (pneumothorax, pneumopericardium and pneumoperitoneum) and in solid organs (including aerobilia and portal venous gas) are beyond the scope of this article; these will be referred only as a cause of abnormal air/gas in the anatomical areas discussed herein.
}

impose prompt diagnosis (which is usually clinic) and treatment, including tracheal intubation and/or emergency tracheostomy.

Imaging studies are helpful to confirm the diagnosis on doubtful cases, exclude local associated complications, determine the extension, and monitor the evolution.

Retroperitoneal causes of ectopic air are hardly ever lifethreatening in themselves, as no vital structures are under concern. Thus, treatment will mainly depend on the type of lesion/injury and the patient's clinical presentation. As a general rule, small perforations that are expected to spontaneously close may be conservatively managed - by "nothing by mouth", intravenous hydration and broad-spectrum intravenous antibiotics, as well as serial imaging studies. Large perforations are indicated for surgical treatment [10].

Surgical procedures are a frequent cause of sparse air bubbles, usually in the proximity of the intervention site. The complete absorption of ectopic gas often occurs within 48$72 \mathrm{~h}$, thus conservative-expectant management is the option, if the patient is otherwise asymptomatic. Serial imaging studies are performed when patients are suspected to have any early postoperative complication; evidence of increased ectopic gas/ air is worrisome and may be related to an anastomotic leak or infectious complication. In the era of laparoscopic surgery, one should be aware that insufflated gas (usually carbon dioxide) is sometimes temporarily retained within the abdomen, and may cause transient upper abdominal and shoulder pain, which may persist for about 3 days $[6,11]$.

Gas-forming infections are a special matter of concern; clinical outcome will largely depend on a prompt diagnosis and treatment. Thus, imaging plays an important role to achieve the correct diagnosis and extension of the disease. Generally, successful treatment requires aggressive management of the infection with systemic antimicrobial therapy and control of septic shock. Most of the time, these patients are candidates for surgical interventions such as debridement or percutaneous drainage in emphysematous pancreatitis and surgical/percutaneous drainage or even nephrectomy in refractory emphysematous pyelonephritis. Patients with necrotising fasciitis are early submitted to surgical debridements of the affected areas in order to remove all non-viable tissue and help relieve the cause of systemic toxicity [1].

\section{Cervical space}

Local causes of cervicofacial emphysema

1. Loss of oral cavity or pharyngeal mucosal integrity. Sources might be related to endoscopic and surgical procedures (such as teeth extraction, oral, maxillary and facial procedures, and more rarely tonsillectomy) and foreign body traumatism. 

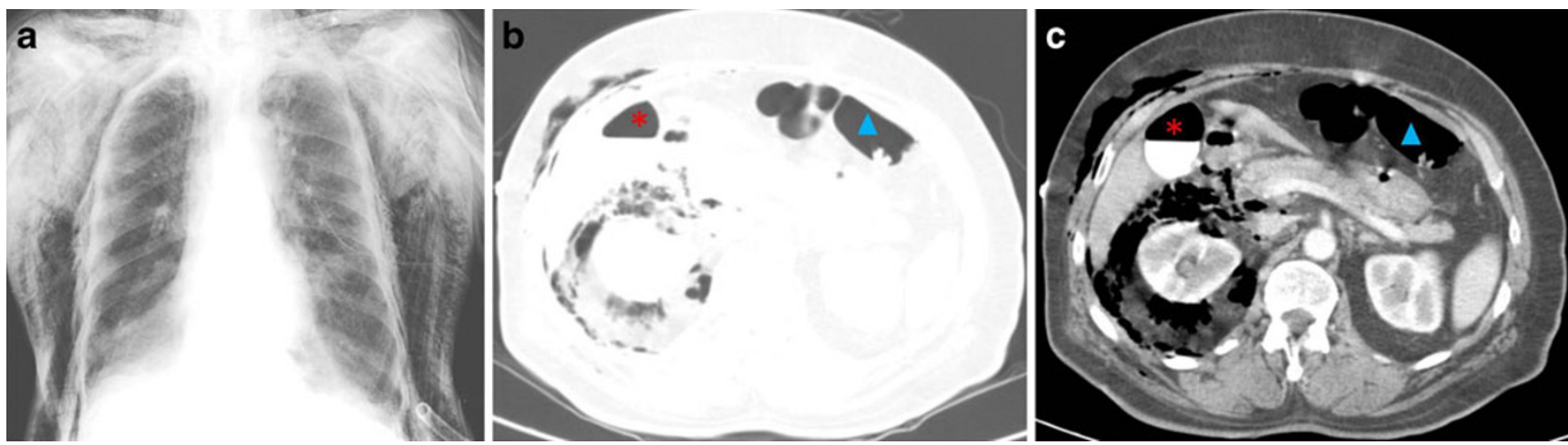

Fig. 1 Imaging subcutaneous emphysema. Duodenal perforation complicating ERCP in a 75-year old woman. a Chest conventional PA radiography shows linear lucent areas in subcutaneous and chest wall tissues. b, c Contrast-enhanced CT scan at the level of the kidneys. The "lung window"

(a) easily depicts the presence of air dissecting the retroperitoneum as well

2. Increased pressure on air pathways, such as excessive coughing, sneezing, vomiting and nose blowing.

3. Trauma to any of the air-containing structures such as paranasal sinuses or tracheal rupture.

4. Soft tissue infections that may arise in the scalp, paranasal sinus, face, oral cavity (e.g. dental abscess) or neck. Necrotising fasciitis is an aggressive infection complicated by gangrene that quickly diffuses through fascial planes; it is usually related to odontogenic infections in immunocompromised patients, though it has also been described after trauma and tonsillectomy.

\section{Cervical anatomy}

Two major "fascial" layers enclose infrahyoid neck structures: the former superficial fascia, according to Terminologia Anatomica (1998) now known as subcutaneous layer (tela subcutanea) and the (deep) cervical fascia. The tela subcutanea is a fat-filled layer of connective tissue that surrounds the neck and contains the platysma, superficial lymph nodes, nerves and vessels. It allows the skin to as extending to the abdominal wall; the "soft tissue window" (b) accurately localizes ectopic air/gas in the different planes of retroperitoneum, and in the abdominal muscular and subcutaneous tissue. Air is also seen in the contrast filled gallbladder (red star) with an air-fluid level (ERCP-related findings) and transverse colon (blue triangle) easily glide over deeper structures and extends all over the body $[12,13]$.

Although some controversy exists in the description/ nomenclature of the (deep) cervical fascia, for an easy understanding, it is commonly accepted that it consists of three layers: superficial, middle and deep layers, which divide the deep neck into multiple spaces (Fig. 3).

The superficial (or investing) layer lies between the superficial cervical fascia and the muscles of the neck; it splits to encircle the sternocleidomastoid and trapezius muscles, to blend posteriorly with the ligamentum nuchae, and splits again between the angle of mandible and the mastoid process to enclose the parotid gland. The middle fascia extends from the skull base superiorly to the mediastinum inferiorly and anteriorly from the hyoid bone to thoracic inlet; it is divided into muscular and visceral divisions (also called pretracheal and buccopharyngeal, in the suprahyoid neck), surrounding the strap muscles and the visceral structures of the neck, respectively. The deep layer of cervical fascia surrounds the deep muscles of the neck and the cervical vertebrae; it extends from the skull base into the mediastinum and has two

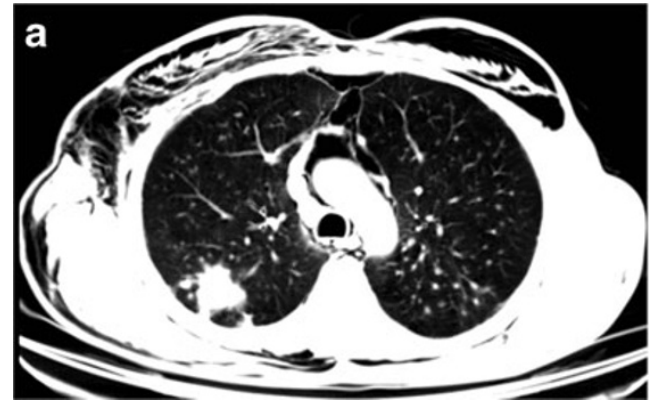

Fig. 2 A 44-year-old man with silicosis and CPOD (chronic pulmonary obstructive disease), with an upper right lobe nodule, who developed secondary pneumothorax after biopsy; the lung did not expand with drainage, due to the development of broncho-pleural fistula; the drain

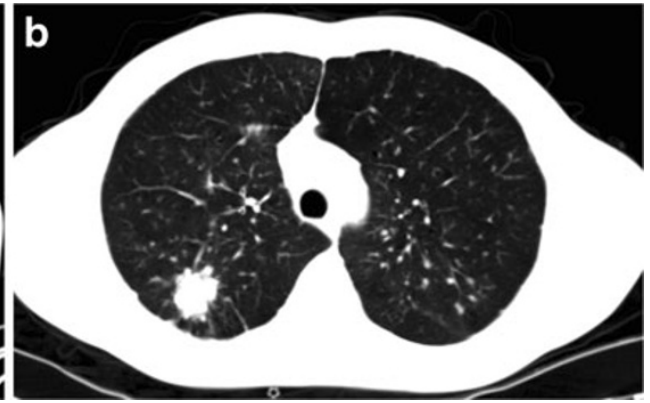

was accidentally removed and the patient developed rapidly progressive emphysema. a Axial CT scan in lung windows depicts extensive subcutaneous emphysema and pneumomediastinum. b Axial CT scan 5 days later, shows that all ectopic air has been reabsorbed 

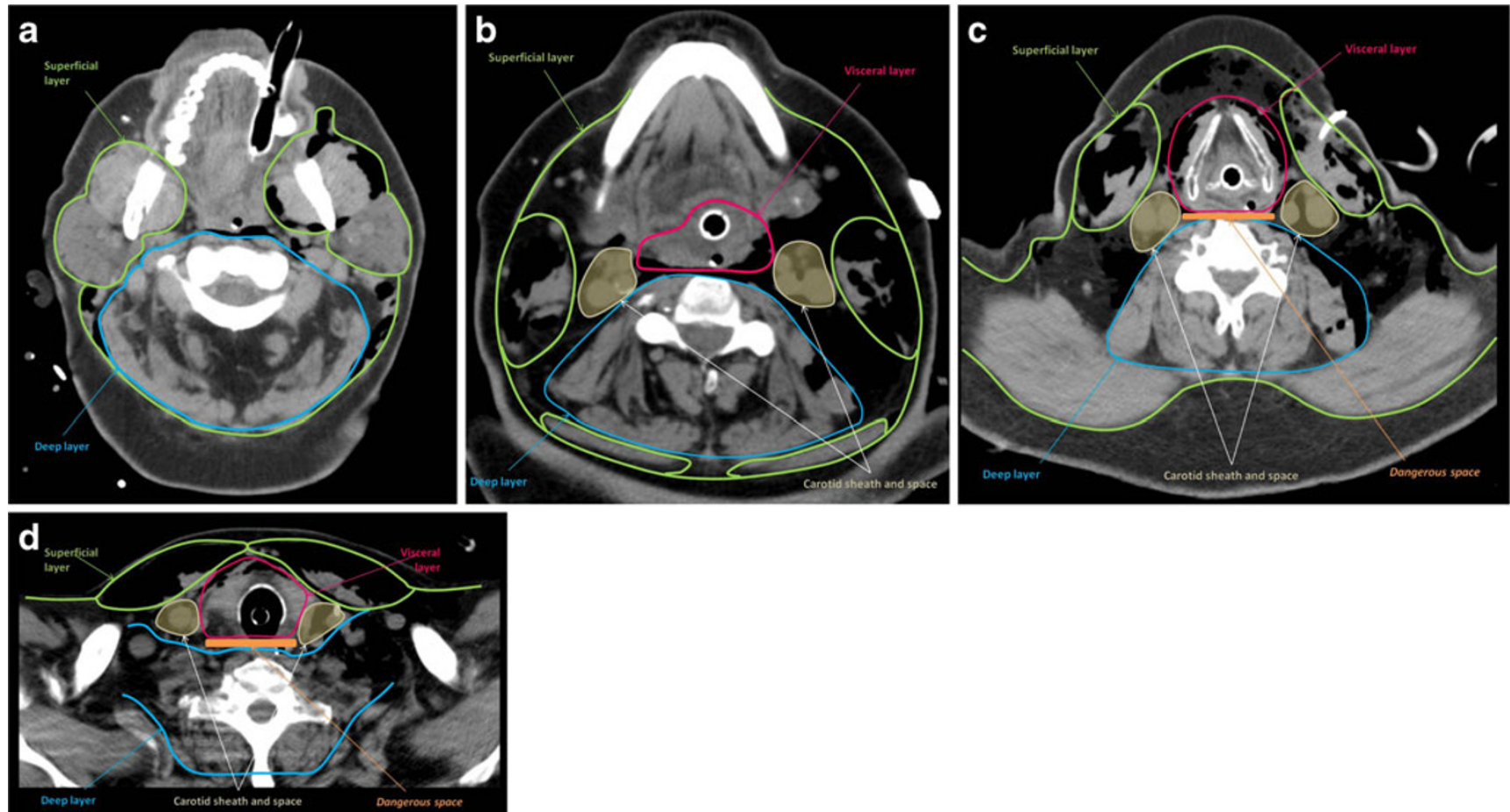

Fig. 3 Cervical emphysema in a 63-year-old man with tracheal rupture after endotracheal intubation (respiratory failure in CPOD patient complicated by lung infection). Contrast-enhanced CT scan at supra-hyoid (a and $\mathbf{b}$ ) and infra-hyoid (c and d) cervical levels, illustrate the cervical fascia and its layers. The superficial or investing layer (green line) is superiorly attached to the superior nuchal line of the occipital, the mastoid process of the temporal bone and the inferior border of the mandible, and inferiorly to the manubrium, clavicles and scapula; laterally it merges with subcutaneous tissues of the neck. The middle fascia (pink line) runs, anteriorly, from the hyoid downwards in front of the trachea and large vessels, ultimately blending with the fibrous pericardium; and posteriorly

divisions: the alar and the prevertebral layers; the alar layer forms the posterior and lateral wall of the retropharyngeal space, and bridges the transverse processes of the vertebrae; the prevertebral layer encloses the paraspinal muscles. The alar layer is attached to the prevertebral layer by loose connective tissue only, and thus an easily distended space is found between them. This space is limited above by the base of the skull, while below it extends behind the oesophagus into the posterior mediastinum to the diaphragm, creating a conduit for free movement of air/gas between mediastinum and neck. This space has been named "dangerous space", as it allows the spread of the infection between the neck and mediastinum. The prevertebral layer laterally forms a sheath for the brachial nerves and subclavian vessels in the posterior triangle of the neck, and it is continued under the clavicle as the axillary sheath $[6,12,14,15]$ (Fig. 3c and d).

So, the layers of the cervical fascia define important spaces that limit air spread along cervical tissues:

1. Pretracheal space. The space anterior to the trachea and posterior to the strap muscles and pretracheal fascia. Its

from the skull base, attaches to the prevertebral fascia, and merges with the investing fascia at the lateral borders of the infrahyoid muscles. The deep or prevertebral layer (blue line) encircles the paraspinous and perivertebral muscles; it runs from the skull base downwards to the coccyx; its more anterior layer contribute to the posterior and lateral wall of the retropharyngeal space and insert on the diaphragm; it gives off a thin lamina, the alar layer, attached to the prevertebral layer by loose connective tissue, creating a potential space between mediastinum and neck, the dangerous space (orange shaded area) - for free movement of air/gas between mediastinum and neck

upper limit is bound by the thyroid cartilage and below in the mediastinum by the pericardium and parietal pleura at the level of the carina.

2. Visceral space. As suggested by the name, this infrahyoid space encloses the thyroid and parathyroid glands, larynx and trachea, and pharynx and oesophagus. This space continues into the chest, creating a conduit of free movement of air/gas between the neck and the mediastinum [2].

3. Carotid space. This space is surrounded by the carotid sheath. It is formed by the fusion of the major layers of cervical fascia and contains the carotid artery, internal jugular vein and vagus nerve, and descends into the chest with these structures.

4. Retropharyngeal space. Fat-filled space between the middle layer of cervical fascia anteriorly and the alar layer of the deep layer posteriorly and laterally; extends from the skull base to the level of the T4 vertebral body, and inferiorly it connects with the danger space.

5. Perivertebral space. Enclosed by the prevertebral fascia, this space is continuous from the skull to the coccyx. 
Air/gas spread to the upper limbs

The superficial cervical fascia laterally merges with subcutaneous tissues of the neck.

The cervicoaxillary canal with its fibrous neurovascular sheath, enclosing the first part of the axillary artery, the axillary vein and the brachial plexus, forms a passageway between the neck and the upper limb, also allowing air/gas to go through.

The muscular fascia also enables air/gas spread between the trunk and the upper limbs, as happens with the upper limb muscular insertions of scapular girdle, and the extensions of fascia in between (e.g. the floor of the axilla is formed by the fascia extending among the pectoralis major and the latissimus dorsi) [16].

Where is the connection between neck and chest?

There are no barriers on the subcutaneous tissues all over the body. So, free movement of air/gas is possible just under the skin. An equivalent free air/gas flow is possible in the perivertebral space. The visceral and retropharyngeal spaces directly communicate with the mediastinum, creating a conduit for free movement of air/gas between mediastinum and neck [2] (Fig. 4).

\section{Mediastinal space}

A pathological accumulation of air/gas in mediastinum is called pneumomediastinum.

\section{Local causes of pneumomediastinum}

1. Large airways: mainly related to traumatic events.
2. Small airways: whenever the pressure gradient between the air-filled alveoli and the surrounding interstitium increases, it might be sufficient to cause alveolar rupture. Air then spreads along the perivascular and peribronchial fascial sheaths and reaches the mediastinum-the Macklin effect (Fig. 5a and b). This may occur in the setting of: (a) air trapping due to airway narrowing or mucous plugging (e.g. asthma), (b) straining against a closed glottis (e.g. forced coughing, parturition, and weight-lifting), (c) blunt or barotrauma, including vigorous cardiopulmonary resuscitation $[2,17]$.

If under high pressure (because of an abrupt increase in pressure or insufficient decompression into cervical and subcutaneous tissues and/or to retroperitoneum), pneumomediastinum may lead to rupture of the mediastinal pleura, with air decompressing to the pleural cavity. Nevertheless, the opposite is not true-pneumothorax does not lead to pneumomediastinum in normal circumstances [2]. Although it is difficult to prove this sequence by imaging, it is important to be aware that one of the potential complications following alveolar rupture is pneumothorax. Once pneumothorax is established, if the patient is on positive-pressure ventilation it should be withdrawal, if feasible, and a tension pneumothorax should be considered - a life-threatening condition that requires immediate drainage [2].

3. Oesophagus: oesophageal perforations are full-thickness wall tears that may have several aetiologies: (a) iatrogenic (e.g. endoscopic procedures, nasogastric tube placement, endotracheal tube misplacement, thoracic surgery; (b) forceful emesis (Boerhave syndrome); (c) oesophageal inflammatory and neoplastic diseases; (d) postoperative status (oesophageal anastomosis); (e) caustic injury; (f) foreign bodies.
Fig. 4 The same patient as in Fig. 3: tracheal rupture after endotracheal intubation. Sagittal (a) and coronal (b) reformatted CT scan illustrate cervico-thoracic continuum through retropharyngeal (orange) and visceral (in between pink lines) spaces
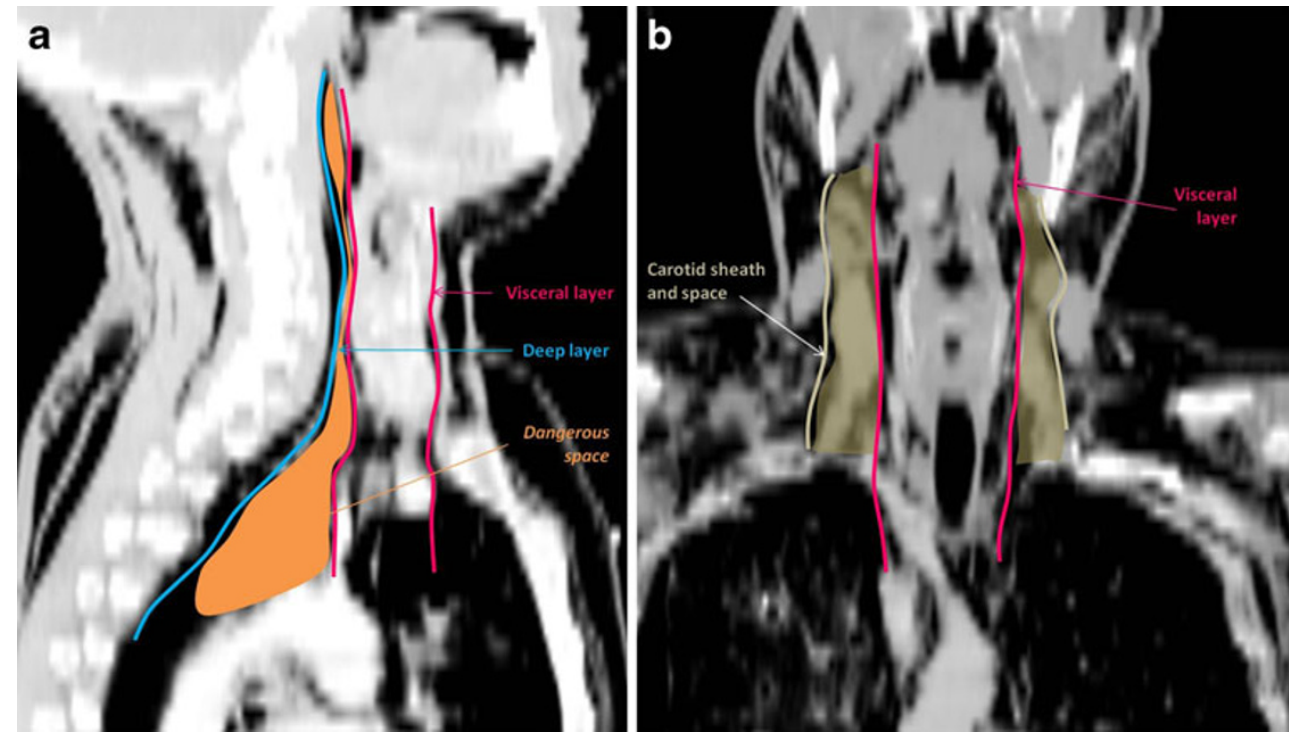


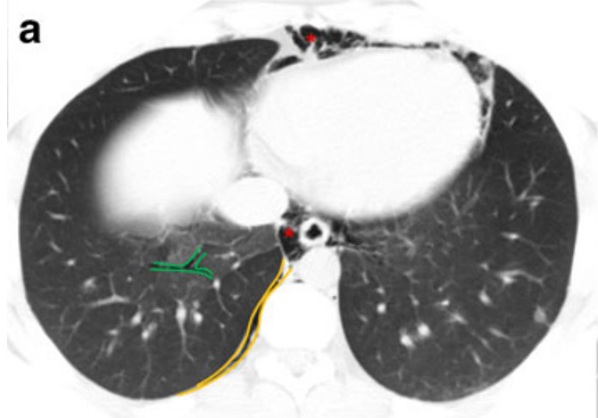

b

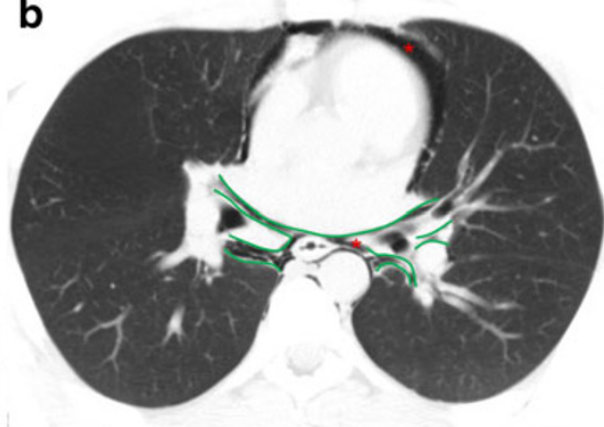

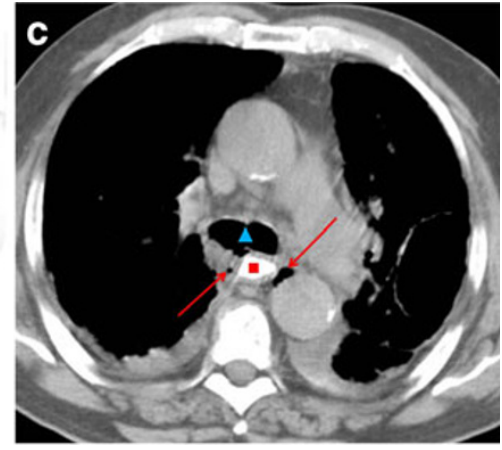

Fig. 5 Local sources of pneumomediastinum. a, b Spontaneous pneumomediastinum due to prolonged and forceful Valsalva manoeuvres in 21-year-old man. Axial CT scan with "lung window" demonstrates the Macklin effect: air is seen along the perivascular and peribronchial fascial sheaths (a), continuous to the mediastinum (red star) (b). c Oesophageal perforation by fishbone complicated by mediastinitis in a 61 -year-old

Once an oesophageal perforation occurs, along with air, contaminated saliva and gastric contents, especially bile and strong acids enter the mediastinum, resulting in mediastinitis. On CT, densification of the mediastinal fat along with air/gas is highly suspicious of mediastinitis, a life-threatening septic condition, which requires urgent intervention and intensive care unit admission (Fig. 5c).

\section{Mediastinal anatomy}

According to Terminologia Anatomica the fasciae of the trunk are listed as parietal, extraserosal and visceral. The fascia parietalis is defined as a sheet or band of fibrous tissue which lies outside the parietal layer of a serosa and lines the wall of a body cavity; the parietal fascia of the thorax is the endothoracic fascia and that of the abdomen is the endoabdominal fascia. The endothoracic fascia is firmly attached to the costal pleura, and posteriorly blends with the prevertebral fascia [18].

The mediastinum lies between the right and left pleura; it extends anteriorly from the sternum (undersurfaced by the endothoracic fascia), to the vertebral column posteriorly (prevertebral fascia), and contains all the thoracic viscera except for the lungs. Despite being academically divided into upper, anterior, middle and posterior compartments, it represents a single space and thus air/gas freely moves inside (Fig. 6).

Where is the link between chest and abdominal cavity?

During the early embryonic life, the coelomic cavity is formed, lined by a serous membrane, and deep to it lies the subserosal space; the integrity of this space is maintained during the division of the coelomic cavity, forming a thoracoabdominal continuum, which crosses the diaphragmatic hiatuses connecting the subperitoneal and subpleural portions of the subserous space [19, 21] (Fig. 7). woman. Axial CT scan after oral iodinated contrast ingestion, distending the oesophagus (red square); extraluminal air bubbles (red arrows) are seen in mediastinum together with increased mediastinal fat attenuation, as well as multiple reactive lymph nodes. Normal permeable airways are seen (blue triangle) at level of carina

The parietal pleura is attached to the thoracic wall by the endothoracic fascia. This fascia lines the diaphragm superiorly, while the transversalis fascia, which is the innermost layer of the endoabdominal fascia, lines its inferior surface [18]. The costal insertions of the diaphragm on the inner surfaces of the lower six ribs and their cartilages provide a separation between theses sleeves. However, three anterior defects in the diaphragmatic attachments provide blending of the two fasciae: [1] one in the midline, between the two slips of sterna origin of the diaphragm that arise from the back of xiphoid process and [2] two parasagittal gaps between the sternal and costal origins of the diaphragm - the sternocostal triangles or foramen of Morgagni, through which run internal mammary vessels that continue in the abdomen as the superior epigastric vessels (Fig. 7a and b). Therefore, air/disease processes that follow these diaphragmatic defects may enter a space between the transversalis fascia and the parietal peritoneum, the properitoneal (or preperitoneal) space, and spread through it laterally to the flank, arriving into the retroperitoneum[18, 20, 21].

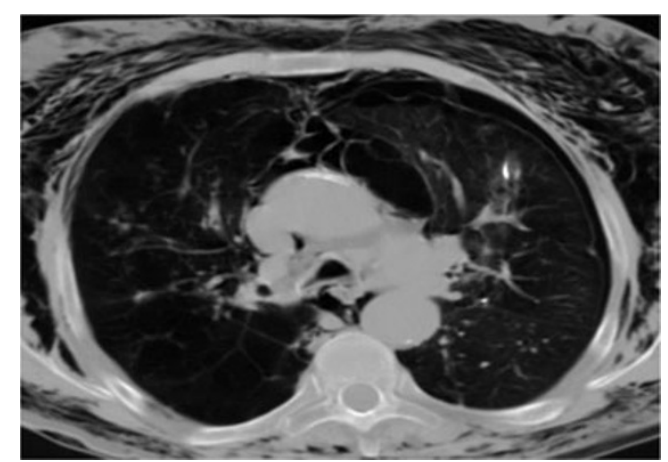

Fig. 6 Unilateral secondary spontaneous pneumothorax in a 69-year-old man with silicosis, and extensive emphysematous changes in lungs, with bullae. Axial CT scan depicts air spread in the mediastinum as a continuum space, and subcutaneous planes after percutaneous drainage of pneumothorax (chest tube not shown) 

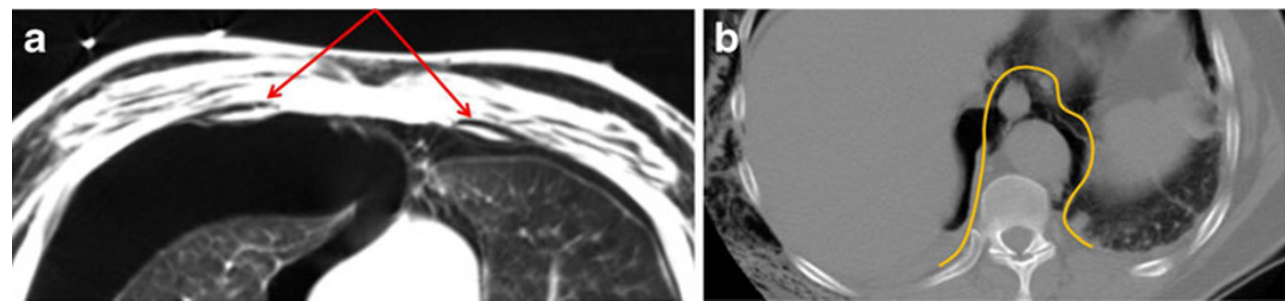

Fig. 7 The same patient as in Fig. 2. Axial CT in the "lung window" depicts the thoracoabdominal continuum. a The anterior blending of the endothoracic and endoabdominal fascias outlined by air: one in the midline, between the two slips of sternal origin of the diaphragm on the back of xiphoid process, and two parasagittal between the sternal and

Posteriorly, endothoracic and transversalis fasciae are continuous behind the diaphragm, at lumbocostal arches and the aortic and oesophageal hiatus, providing communication point between mediastinum and retroperitoneum (Fig. 7b) [21].

Besides these fully accepted congenital communications, there are also areas of structural weakness of embryologic origin, as tiny focal defects on the tendinous part of the diaphragm or the rare septum transversum lacunar aplasia, which allows transphrenic migration of air/gas [19].

The vena caval foramen is not considered part of the thoracoabdominal continuum because the wall of this vessel sticks to the margins of the foramen and thus interrupts continuity of the subserous space [20].

Thus, subserous space provides not only avenues through which vital structures come from thorax to abdomen and vice versa but also pathways for bidirectional spread of disease processes [20].

\section{Retroperitoneal space}

As in mediastinum, a pathological accumulation of air/gas in retroperitoneum is called pneumoretroperitoneum.

\section{Local causes of pneumoretroperitoneum}

1. Retroperitoneal hollow viscera rupture, non-traumatic (e.g. perforated duodenal ulcer, diverticulitis, perforated carcinoma, foreign body perforation) or traumatic (e.g. endoscopic evaluation of the gastrointestinal tract, endoscopic retrograde cholangiopancreatography [ERCP] with/ out biopsy, surgery, blunt or penetrating abdominal trauma).

2. Emphysematous infections (e.g. emphysematous pancreatitis, which represents a superimposed infection with gas-forming bacteria in previously acute pancreatitis, and may also be a sign of end-organ infarction; emphysematous pyelonephritis, usually associated with poorly controlled diabetes mellitus, both life-threatening conditions, with poor prognosis) (Fig. 8) [1].

3. Residual air from retroperitoneal surgery (e.g. urological/ adrenal, anterolateral approach of spinal surgery). costal origins of the diaphragm - the sternocostal triangles or foramen of Morgagni. b The crura have separated, forming the oesophageal hiatus. The subpleural space of the thorax and subperitoneal space of the abdomen are in continuity through the subserous space within the oesophageal and aortic hiatus

\section{Retroperitoneal anatomy}

The retroperitoneum is the space between the posterior parietal peritoneum and the transversalis fascia, and extends from the diaphragm superiorly to the pelvis inferiorly. Although traditionally divided in three distinct compartments, the anterior pararenal, the perirenal and the posterior renal spaces, by the anterior (Gerota or Toldt) and the posterior (Zuckerlandl) renal fasciae, more recent work has demonstrated that the renal fascia is a single multilaminated structure, which contains potential space, named the interfascial plane; these potential spaces are represented by the retromesenteric, retrorenal, lateroconal and combined interfascial planes [22-24] (Fig. 9).

The anterior pararenal space (APS) is bounded anteriorly by the posterior parietal peritoneum, laterally by the lateroconal fascia and posteriorly by the anterior renal fascia; it contains the pancreatic gland, retroperitoneal segments of the duodenum, ascending

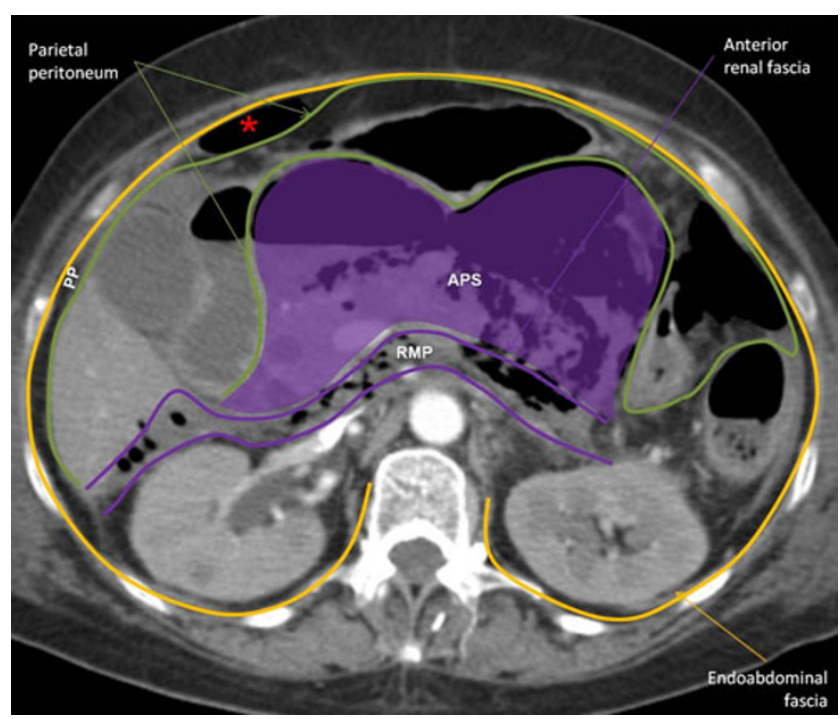

Fig. 8 Emphysematous necrotising pancreatitis in a 64-year-old woman. Contrast-enhanced axial CT scan demonstrates a mottled collection of gas bubbles mainly involving the body and tail of the pancreas; inflammatory changes in the surrounding fat are seen. Gas spread is depicted along the retromesenteric plane and along properitoneal space (red star). RMP retromesenteric plane, $A P S$ anterior pararenal space, $P P$ properitoneal space 
and descending colon and the mesenteric root, as well as adipose tissue. This space is continuous across the midline [25].

The posterior pararenal space (PPS) is bound anteriorly by the posterior renal fascia and posteriorly by the transversalis fascia, and it only contains adipose tissue, lymphatics and blood vessels. Medially, it is limited by the margin of the psoas muscle. The posterolateral portion of PPS has anatomic continuity with the properitoneal flank "stripe" (a layer of areolar tissue and variable amount of fat in between the transversalis fascia and parietal peritoneum) Therefore, air arising within or entering this space may spread to the anterior abdominal wall. PPS also continues superiorly as a thin subdiaphragmatic layer of extraperitoneal fat $[18,19,25,26]$.
The perirenal space (PS) lies between the anterior and posterior renal fascia; it is a paired inverted cone shaped of tissue containing the kidney, the proximal portion of the collecting system, renal and perirenal vasculature, perirenal lymphatics, adrenal glands and variable amount of fat. The space is divided into multiple compartments by thin fibrous lamellae, which form bridging septa crossing the perinephric fat and connect the renal capsule and the anterior and posterior renal fascia, providing a bidirectional channel between spaces [24] (Fig. 10a).

There is a considerable disagreement with the exact arrangement of the renal fascia. In Meyers' concept the renal fasciae are closed above and below the kidney and adrenal
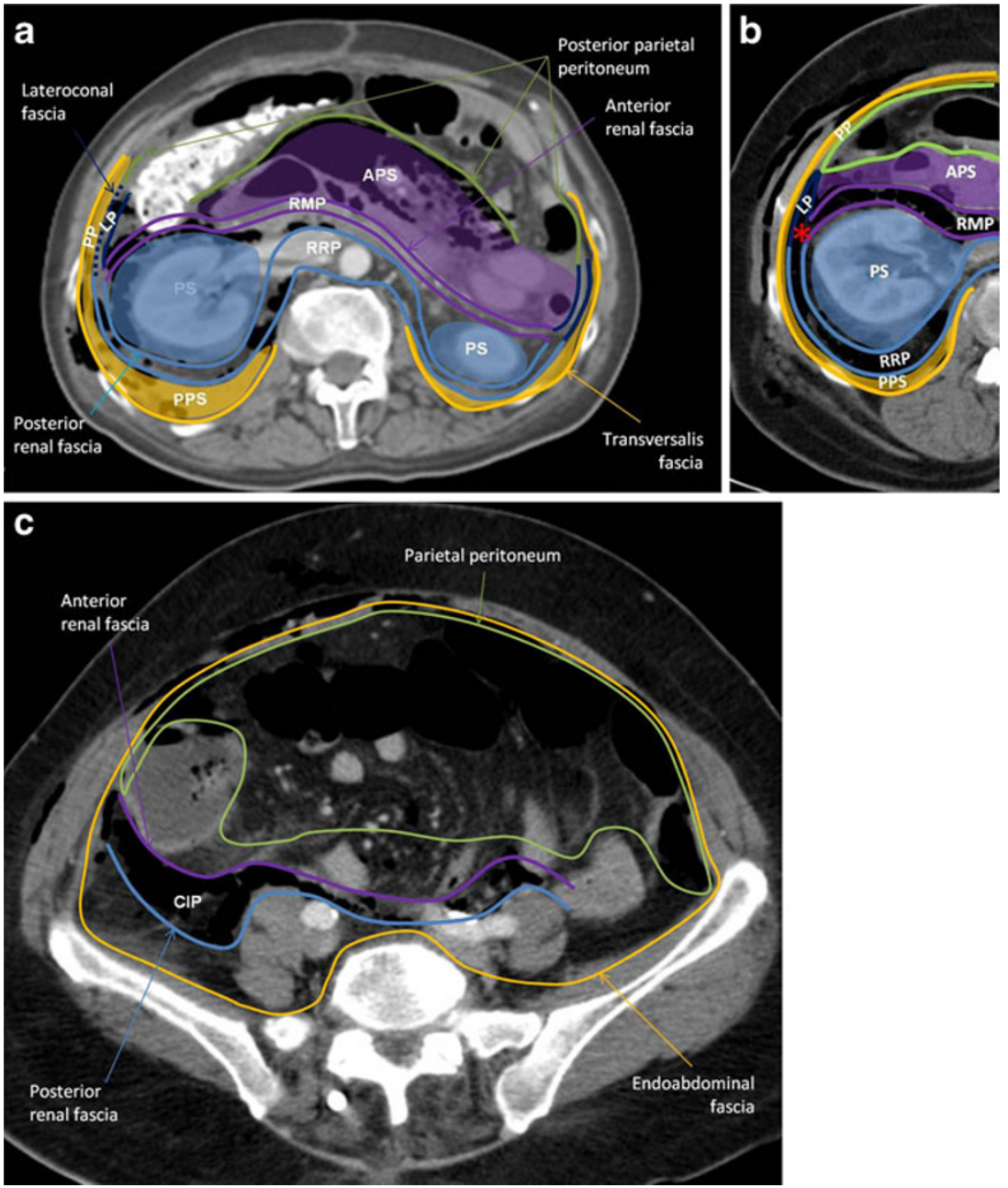

Fig. 9 Duodenal perforation complicating ERCP in a 60-year-old man. Contrast-enhanced CT scan at the level of right renal hilum $(\mathbf{a}, \mathbf{b})$ and iliac crest (c) shows the retroperitoneal and interfascial planes. a Anterior pararenal space (APS) is limited by posterior parietal peritoneum and anterior renal fascia, with midline continuity; posterior pararenal space (PPS) between posterior renal fascia and transversalis layer of endoabdominal fascia; perirenal space (PS) between anterior and posterior renal fascias. Renal and lateroconal fasciae are laminated, defining potential spaces: the retromesenteric (RMP), the retrorenal (RRP) and lateroconal planes (LP) that all communicate at fascial trifurcation. b Axial image focused on fascial trifurcation. Ectopic gas is seen extending through the retromesenteric plane (RMP), retrorenal plane (RRP) and lateroconal plane (LP), and meeting at the fascial trifurcation (red star). Posterior pararenal space (PPS) anteriorly continues as a fat stripe in the properitoneal space (PP). c Inferior extension of the interfascial plane, delineated by ectopic gas/air. The retromesenteric and the retrorenal planes approximate one another as the fat cone of perirenal fat diminishes inferiorly, resulting in the combined interfascial plane (CIP); it continues in the pelvis along the anterolateral margins of the psoas muscle contiguous with the pelvic retroperitoneal perivesical and presacral spaces 

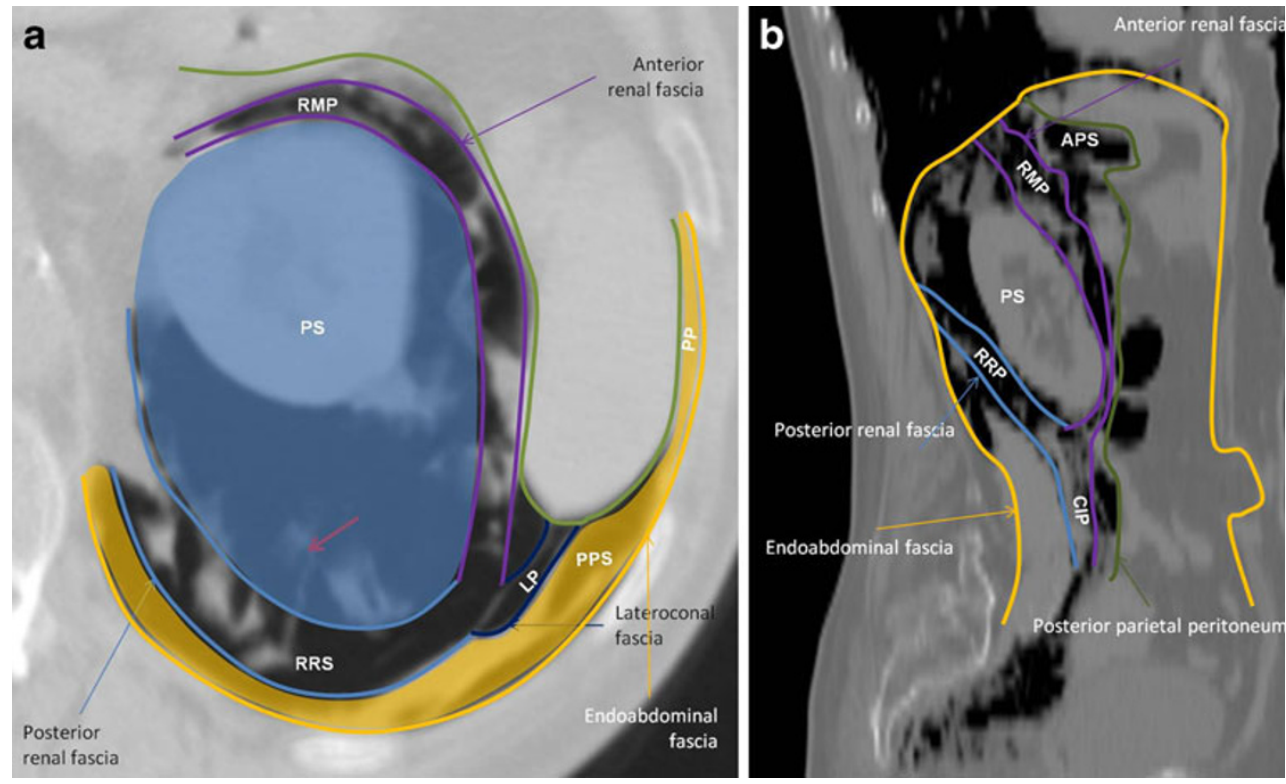

Fig. 10 Sigmoid perforation during colonoscopy with polyp excision in a 77-year-old woman. Axial (a) and coronal (b) reformatted CT scan in "lung window" demonstrating the retroperitoneal anatomy delineated by air/gas. a Perirenal space limited by the anterior and posterior renal fascias, with bridging septa (red arrow) and adipose tissue. Posterior pararenal space anteriorly continues along the properitoneal flank stripe to the properitoneal space between the parietal peritoneum and the transversalis fascia. b On the left, the retromesenteric plane $(R M P)$ and the perirenal space $(P S)$ extend cranially to the dome of the diaphragm, where they blend with the left subdiaphramatic extraperitoneal space. Incidental anterior abdominal wall hernia. $R R P$ retrorenal plane, $P P R$ posterior pararenal space, $P P$ properitoneal space, $L P$ lateroconal space, $C I P$ combined interfascial plane glands; however, clinical studies have shown fluid collections extending into the adjacent spaces, bare area of the liver on the right, superior extraperitoneal space on the left, towards the midline from each perirenal space and into the lower retroperitoneal space of the abdomen and pelvis [25-27]. So, the concept of a multilayered structure of the fascia, helps to understand these findings.

The retroperitoneal interfascial planes are potential expandable planes; the retromesenteric plane stands between the APS and PS, and is continuous across the midline and in front of the major vessels; the retrorenal plane between the PS and PRS communicates with the vascular compartment of the retroperitoneum, including the aorta and inferior vena cava; the lateroconal interfascial plane is between the layers of the lateroconal fascia, and communicates with the retromesenteric and the retrorenal planes; the combined interfascial plane is formed by the (lower) blending of the retromesenteric and retrorenal planes, inferiorly to the cone of perirenal fat, and posteriorly to the ascending and descending mesocolon, and continues along the surface of the psoas muscle to the pelvis [22].

How does retroperitoneal air/gas reach the extraperitoneal abdominal and pelvic spaces?

Superiorly, the left retromesenteric plane and the PS extend to the dome of the diaphragm, where they connect with the left subdiaphramatic extraperitoneal space [22, 27] (Fig. 10b). On the right the retromesenteric plane extends at the level of the right inferior coronary ligament and the bare area of the liver, and communicates with the liver hilum through the subperitoneal space of the hepatoduodenal ligament (Fig. 11). Inferiorly, the combined interfascial plane continues in the pelvis along the anterolateral margins of the psoas muscle, contiguous with the pelvic retroperitoneal perivesical and presacral spaces [22, 24]. The PPS is in continuity with properitoneal space (Fig. 9).

These paths are also based on the continuity of the transversalis fascia (as the outer border of the retroperitoneal and peritoneal cavities) with the parietal endopelvic fascia, lining the abdominopelvic cavity and outlining a conduit for air/gas spread [2, 24, 28] (Fig. 12).

Thus, air in the retroperitoneum can spread across the midline in the APS and via the anterior interfascial retromesenteric plane, and get off to the pelvic extraperitoneal space through the properitoneal fat and by the combined interfascial plane [24].

\section{Extraperitoneal abdominal and pelvic space}

Local causes of extraperitoneal emphysema (Fig. 13)

1. Hollow viscera rupture (e.g. rectal perforation by diverticulitis, perforated carcinoma, foreign body, post endoscopic evaluation).

2. Penetrating pelvic trauma. 
a

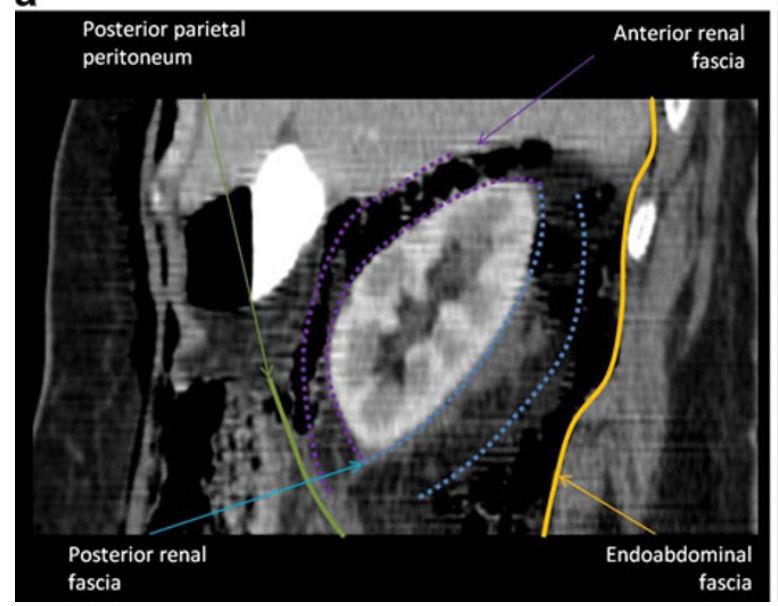

Fig. 11 Duodenal perforation complicating ERCP in a 75-year-old woman. Sagittal reformatted (a) and axial (b) contrast-enhanced CT scan demonstrates the right superior extension of the interfascial plane. The b

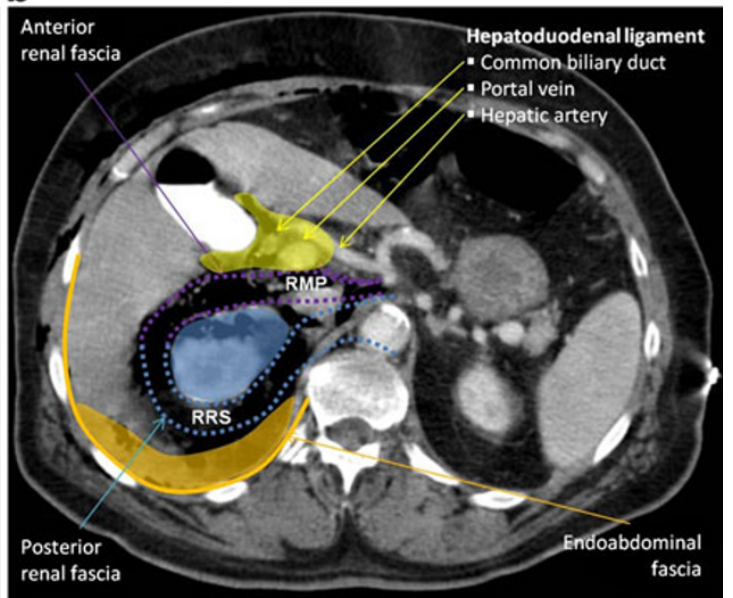

multilaminated retroperitoneum is open towards the upper abdominal extraperitoneal space: the bare area of the liver, and communicates with liver hilum through the subperitoneal space of the hepatoduodenal ligament
3. Residual air from previous surgery (e.g. abdominoperineal amputation, hysterectomy, caesarean).

4. Emphysematous infection: e.g. necrotising fasciitis of perineal, genital or perianal regions (Fournier gangrene) is a polymicrobial infection complicated by thrombosis of small subcutaneous vessels and rapid gangrenous involvement of the surrounding skin and deep fascia; although the diagnosis is based on clinical examination, CT plays an important role not only in confirming the diagnosis, but essentially to assess the extent of disease to plan the surgical treatment, sometimes identifying a potential underlying cause [1].

\section{Extraperitoneal abdominal and pelvic anatomy}

As already stated, the abdominopelvic cavity is entirely lined by the endoabdominal (parietal abdominal) fascia, whose innermost layer is the transversalis fascia; this name is derived from its location between the inner surface of the transverse abdominal muscle and the parietal peritoneum in the anterior and lateral abdominal wall; nevertheless, it also undersurfaces the diaphragm, is the anterior surface of the anterior longitudinal ligament, paravertebral, iliopsoas and internal obturator muscles; moreover, it delineates the lateral walls and diaphragm of the pelvis $[4,18]$.
Fig. 12 Anastomotic colorectal leak during a CT colonoscopy in a 81-year-old woman with a history of excision of recto-sigmoid adenocarcinoma 8 years previously. Coronal (a) and sagittal (b) reformatted CT scan. Continuity of the transversalis fascia with the parietal endopelvic fascia is depicted
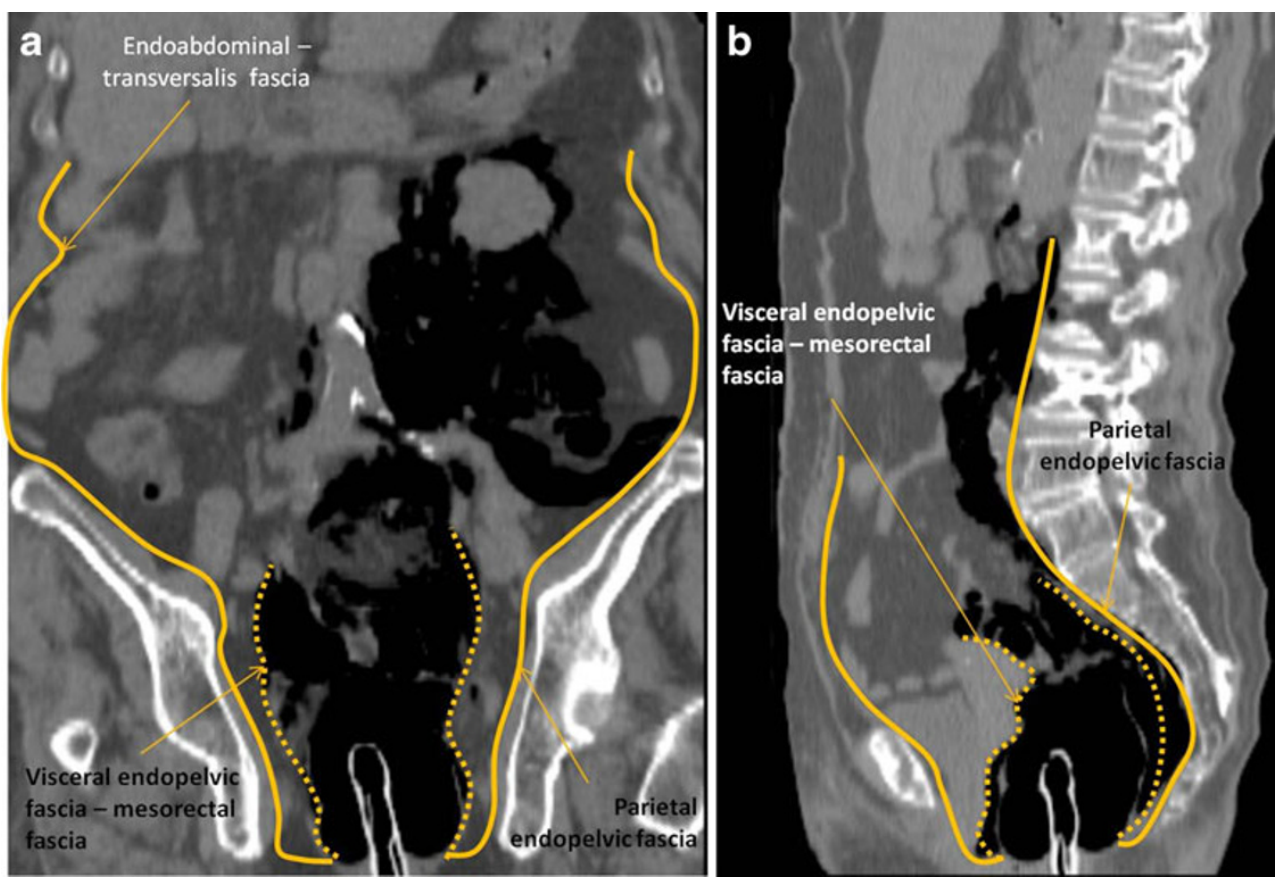


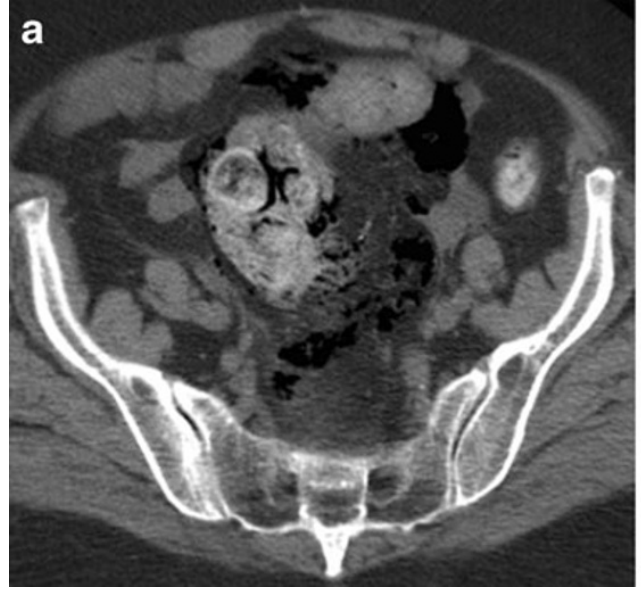

Fig. 13 Local causes of extraperitoneal pelvic emphysema. (a) Sigmoid perforation (necrotising acute colitis probably due to fecaloma) in a 75year-old female. Unenhanced-CT scans of pelvis shows gas pockets in the sigmoid mesocolon, extending superiorly to retroperitoneum through the combined interfascial plane. (b) Fournier gangrene in a 67-year-old

Anatomical and embryological differences dictate that the fascia transversalis should be considered differently from the supra-abdominal and infra-abdominal regions. The arcuate line, which is an horizontal line that demarcates the lower limit of the posterior layer of the rectus sheath, separates the cranial from the caudal part of the abdominal wall; below this line, the aponevrosis of internal oblique, external oblique and abdominal transversal muscles pass anterior to the rectus muscle, and the fascia transversalis becomes stronger and functions as an aponevrosis, attached to the abdominal rectus muscle [18].

In the infraumbilical properitoneal space are the urachus and the obliterated umbilical arteries, which are developmental remnants, forming the medial and the median umbilical ligaments, respectively [18].

The endopelvic fascia is the internal investing fascia of the pelvis and consists of two layers, the parietal layer (how the transversalis fascia is named at this level) and visceral layer; it courses along the lateral borders of the pelvic organs, and encircles the perirectal fat, at this point named mesorectal fascia. Both sleeves are continuous and attached to the diaphragmatic part of the pelvic fascia along the tendinous arch (a thickened band on the upper layer of the diaphragmatic part of the pelvic fascia at the level of a line extending from the lower part of the pubic symphysis to the spine of the ischium) $[14,29]$ (Fig. 12).

The umbilicovesical fascia runs inferiorly from the umbilicus, posterior to tranversalis fascia and anterior to parietal peritoneum, with a triangular configuration with its apex at the umbilicus. It surrounds the urachus and obliterated umbilical arteries. Courses on the pelvis below the peritoneal reflection

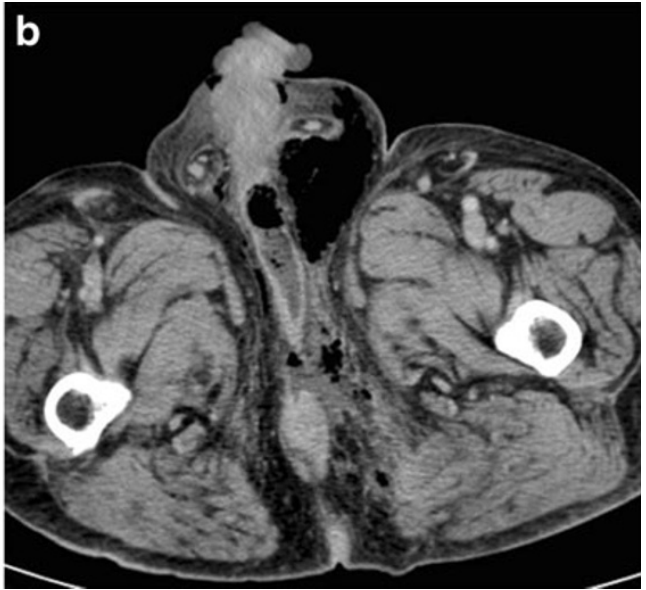

diabetic man. Contrast-enhanced CT scan shows fluid and air pockets tracking in the corpora cavernosa, extending anteriorly to an enlarged scrotal sac containing gas and cranially to the ischiorectal fossa and subcutaneous tissue

and extends to the pelvis floor surrounding the urinary bladder, then blending with the endopelvic visceral fascia along the lateral aspects of the lower uterus or seminal vesicles and the rectum.

Pelvic cul-de-sacs between the rectum and bladder (the rectovesical space in males), and rectum and uterus (the Douglas cul-de-sac in females) are shaped by parietal peritoneum reflections. These reflections extend inferiorly, and their anterior and posterior sleeves are fused, originating septa that separate the urological or genital tract from the rectum: rectovesical and rectovaginal septum.

Thus, a compartmental pelvis may be described as follows $[4,30,31]$ :

\section{Prevesical space}

Anterior and lateral to the umbilicovesical fascia, and posterior to the trasversalis fascia. Prevesical space laterally communicates with the properitoneal space of the abdominal wall and flanks; posteroinferiorly it surrounds the lateral walls of the urinary bladder, and anteroinferiorly it forms the retropubic space (the space of Retzius) [32] (Fig. 11).

2. Paravesical space

Medially is limited by the umbilicovesical fascia, which fuses inferiorly with the visceral sleeve of the endopelvic fascia. The lateral boundary is the parietal endopelvic fascia, and the superior one the peritoneum (Fig. 11).

\section{Presacral space}

Represents the posterior communication between both paravesical spaces. It is located between the rectal and parietal sleeves of the endopelvic fascia (Fig. 11). 


\section{Perivesical space}

Circumscribed by the umbilicovesical fascia anterior and laterally, and rectovaginal/rectovesical septum posteriorly, it encounters the urachus, obliterated umbilical vessels, urinary bladder and the lower uterine segment or seminal vesicles (Fig. 11).

\section{Perirectal space}

Limited by the rectovaginal (in females) or rectovesical (in males) septum, and the visceral endopelvic fascia (rectal fascia) (Fig. 14).

In the abdomen and pelvis, there is another anatomical continuum, the subperitoneal space, deep to the surface lining of the visceral and parietal peritoneum, the omentum and the various peritoneal ligaments and mesenteries, which in turn is an extension of extraperitoneal space, allowing spread of air/gas, fluid and other disease processes [23] (Fig. 13a, b).

\section{Extrapelvic air/gas spread}

The extraperitoneal compartment in the pelvis communicates with the subperitoneal space in the anterior abdominal wall anteriorly and the retroperitoneum posteriorly, easily allowing the spreading of air/gas among them. Besides this, the extraperitoneal pelvis has various outlets for the transit of organs and neurovascular structures to the rest of the body [32] (Fig. 15).

The inferior epigastric vessels courses for a distance in the properitoneal space before entering the rectus sheath just below the arcuate line; thus, air/gas can easily extend along with them, through fascia transversalis, to become intramuscular and rapidly spread interstitially $[4,16,30]$.

Superior and inferior gluteal branches of the internal iliac arteries perforate the pelvic fascia on their way to gluteal region, opening one more channel to air spread [3].

It is also important to recognise that the femoral (vascular) sheath partly consists of a downward prolongation of the transversalis fascia. Consequently, air may extend from the prevesical space (anteriorly limited by the transversalis fascia) to the femoral sheath $[4,30]$.

The inguinal canal is a small passage that leads to the lower abdominal wall. It has openings at either end: the deep and superficial inguinal rings. The deep inguinal ring is a defect in the transversalis fascia that lies laterally to the inferior epigastric vessels. Thus, air contained by this fascia may dissect away through it, alongside the spermatic cord in males and the round ligament in women, respectively until scrotum and grand labia [4, 33].

Fascial investments of some muscles and vessels provide anatomic pathways of spread from the pelvis to the buttocks, hips and thighs. Three pelvic muscles have extra-pelvic insertions: the iliopsoas originates from the transverse processes and bodies of the 12th thoracic and lumbar vertebrae, upper two-thirds of the iliac fossa and sacral ala, inserting on the lesser trochanter of the femur (forming potential pathway of communication between the mediastinum and the upper thigh); the piriformis muscle, which originates from the sacrum and the margin of the greater sciatic foramen and inserts on the greater trochanter of the femur; and the internal obturator, running from the margins of the obturator foramen to insert on the greater trochanter of the femur [3, 32].

Figure 16 summarises the main connections between spaces reviewed.

\section{Conclusion}

Ectopic air/gas represents a challenge to the radiologist. The search for the underlying aetiology may be tricky, since

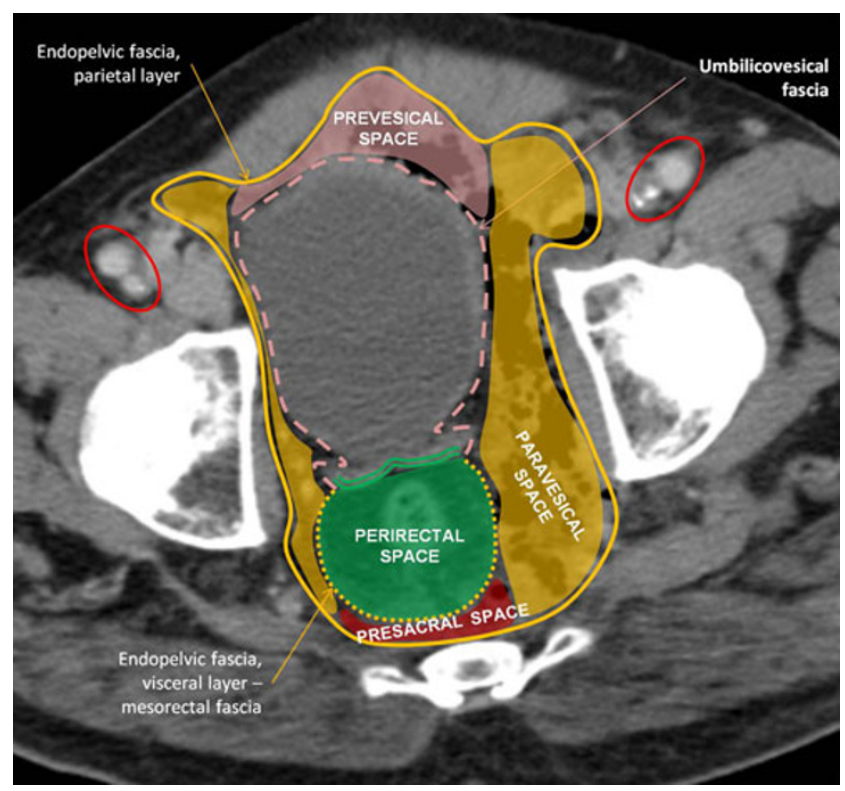

Fig. 14 The same patient as in Fig. 13b. Fournier gangrene in a 67-yearold diabetic man, with extensive arteriosclerosis, already submitted to aortic bifemoral bypass years ago, still permeable (red circles - patent common femoral bypass grafts located anteriorly to native arteries, occluded on the left). Contrast-enhanced CT scan at level of acetabular dome shows endopelvic fascia and extraperitoneal pelvic spaces. Endopelvic fascia and its two layers are illustrated. The parietal layer covers the elevator muscle of anus and coccygeus (pelvic diaphragm) and the intrapelvic portions of the internal obturator and the piriformis muscles. The visceral layer covers inferior segments of the urinary bladder, lower third of the ureters, uterus, vagina and seminal vesicles; it forms a continuous line enveloping the perirectal fat, the mesorectal fascia. Pelvic spaces. Prevesical space is bordered by transversalis fascia anteriorly and umbilicovesical fascia posteriorly. Paravesical and presacral spaces are limited by parietal and visceral sleeves of the endopelvic fascia. Perivesical space is surrounded by umbilicovesical fascia and rectovesical septum. Perirectal space is involved by the rectal fascia and separated from the perivesical space by the rectovesical septum 

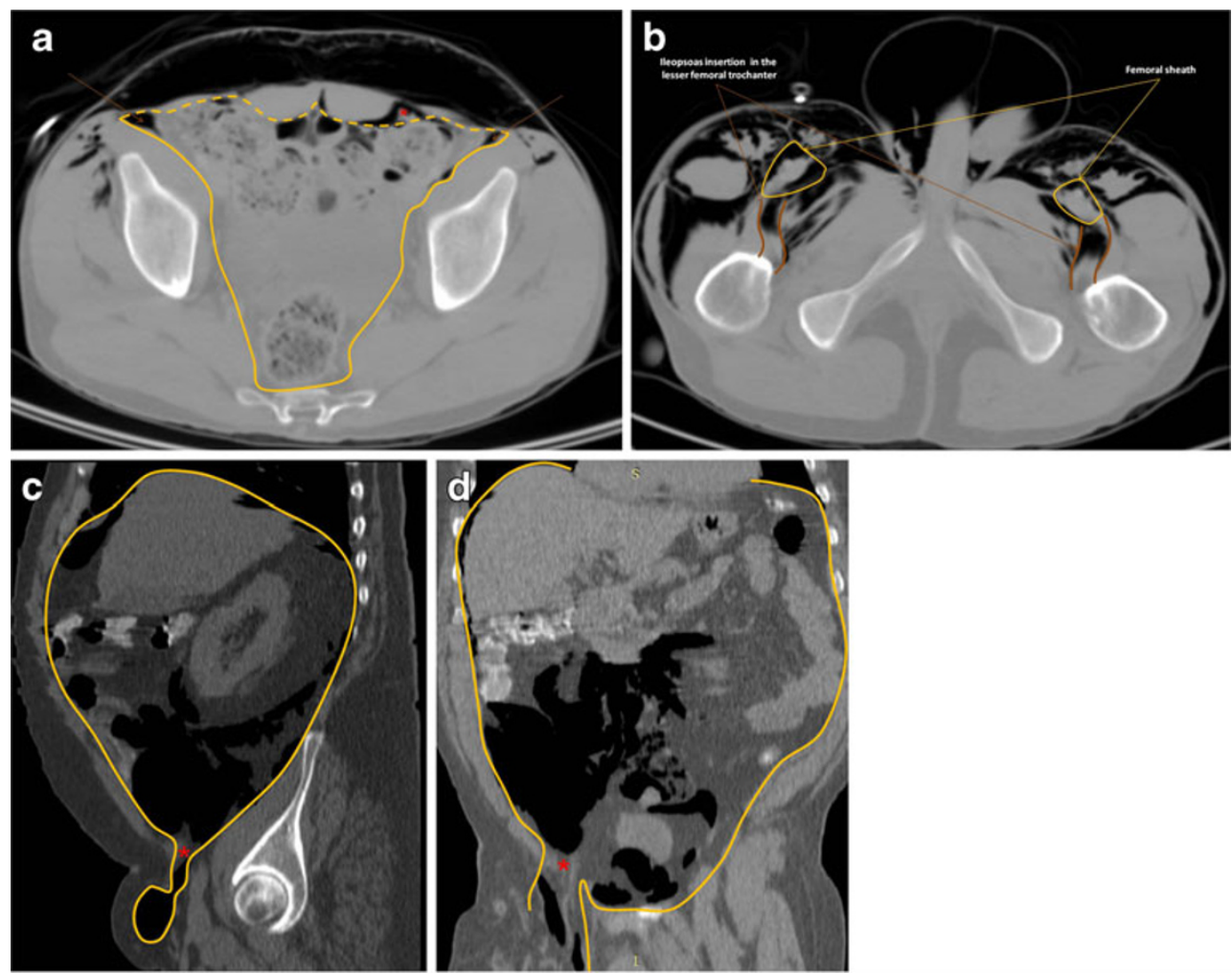

Fig. 15 Air spread from pelvis to inferior limbs. a, b The same patient as in Fig. 2: a 44-year-old man with broncho-pleural fistula secondary to pneumothorax drainage and subsequent rapidly progressive emphysema from neck to groin. a Axial CT in "lung window" shows air spread through the thinned transversalis fascia and abdominal rectus below the level of arcuate line, involving inferior epigastric vessels. Air is seen anteriorly to iliac muscles (brown arrow). b Axial CT caudal to a demonstrates air spreading out the

air can be detected almost anywhere (away from its source). Continuity is described between neck, mediastinum, retroperitoneum and the pelvis on the basis of

pelvis along with iliac vessels through the femoral canal. Air dissecting along muscular fibres and sheaths, as can be seen from the pelvis along iliac muscle until its insertion in the lesser femoral trochanter (brown line). The least resistance of subcutaneous tissues allow easy spread of subcutaneous emphysema. c, d A 60-year-old patient with colonic perforation at optical colonoscopy. Sagittal (c) and coronal (d) reformatted CT images illustrate air spread along the right inguinal (red star) canal and into the scrotal sac

anatomy and fascial-defined spaces; vascular perforations of the fasciae and muscle insertions also constitute potential conduits between compartments.

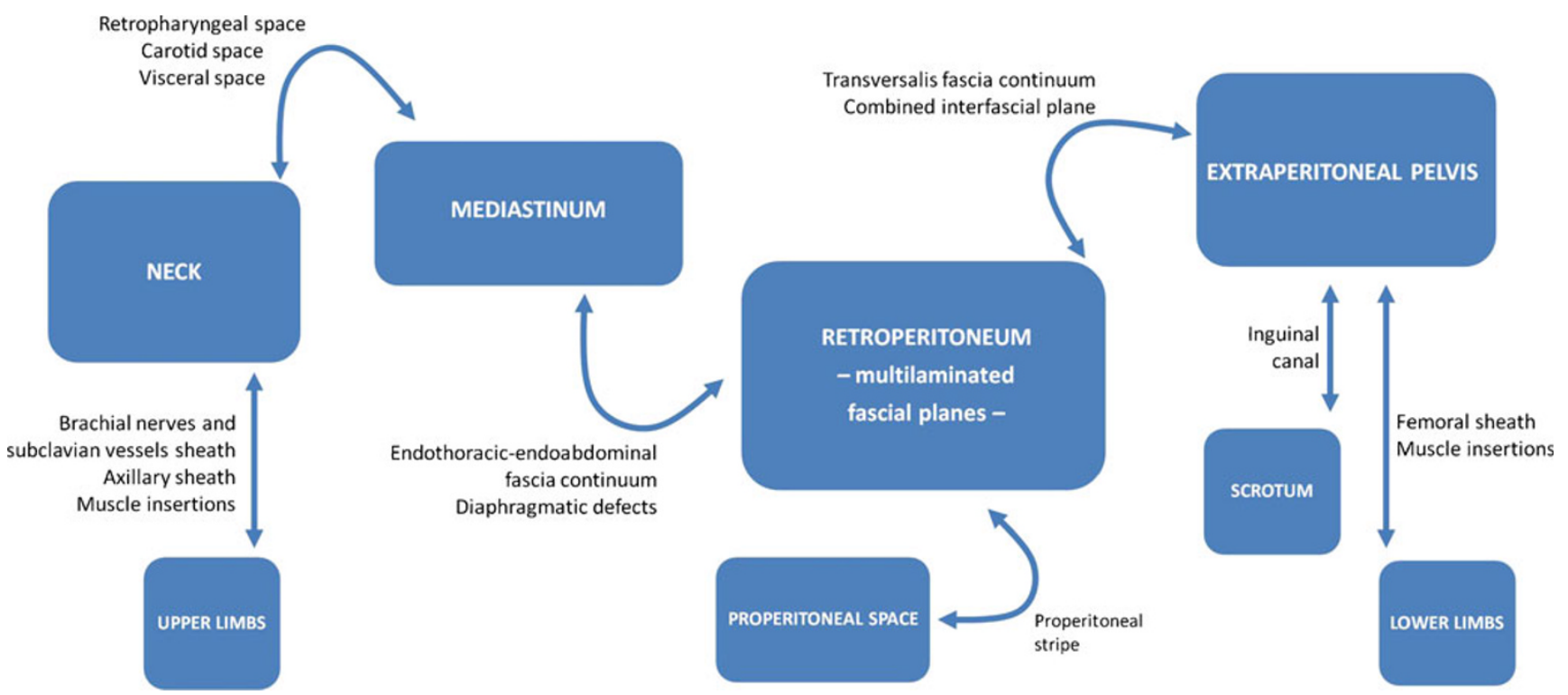

Fig. 16 Ectopic air/gas flow chart through different anatomical spaces 
Acknowledgments Ana Frias Vilaça and Alcinda Reis contributed equally to this work.

Authors thank Liliana Leite for language revision of the article. This work was previously presented as an education exhibit at the 2009 RSNA annual meeting (Space: LL-ER2494).

Disclosures The authors declare no conflicts of interest. No funding was received for this work.

Open Access This article is distributed under the terms of the Creative Commons Attribution License which permits any use, distribution, and reproduction in any medium, provided the original author(s) and the source are credited.

\section{References}

1. Grayson D, Abbott R, Levy A, Sherman P (2002) Emphysematous infections of the abdomen and pelvis: a pictorial review. Radiographics 22:543-561

2. Maunder RJ, Pierson DJ, Hudson LD (1984) Subcutaneous and mediastinal emphysema. Pathophysiology, diagnosis, and management. Arch Intern Med 144:1447-1453

3. Meyers M, Goodman K (1975) Pathways of extrapelvic spread of disease: anatomic-radiologic correlation. AJR Am J Roentgenol 125: 900-909

4. Mastromatteo JF et al (1997) Communications of the pelvic extraperitoneal spaces and their relation to the abdominal extraperitoneal spaces: helical CT cadaver study with pelvic extraperitoneal injections. Radiology 202:523-530

5. Srinivas R, Singh N, Agarwal R, Aggarwal AN (2007) Management of extensive subcutaneous emphysema and pneumomediastinum by micro-drainage: time for a re-think? Singapore Med J 48:e323

6. Prokop M, Galanski M (2003) Spiral and multislice computed tomography of the body. Thieme, Stuttgart New York

7. Williams DJ, Jaggar SI, Morgan CJ (2005) Upper airway obstruction as a result of massive subcutaneous emphysema following accidental removal of an intercostal drain. Br J Anaesth 94(3):390-392

8. Sucena M, Coelho F, Almeida T, Gouveia A, Hespanhol V (2010) Massive subcutaneous emphysema - management using subcutaneous drains. Rev Port Pneumol 16:321-329

9. Fortes F, Sennes LU, Fortes FSG, Imamura R, Tsuji DH (2007) Cervical emphysema as an early complication of tonsillectomy. Arq Int Otorrinolaringol 11:65-69

10. Fujii L, Lau D, Fleischer DE, Harrison ME (2010) Successful nonsurgical treatment of pneumomediastinum, pneumothorax, pneumoperitoneum, pneumoretroperitoneum and subcutaneous emphysema following ERCP. Gastroenterol Res Pract 2010: 289135

11. Tsai HW, Chen YJ, Ho CM et al (2011) Maneuvers to decrease laparoscopy-induced shoulder and upper abdominal pain a randomized controlled study. Arch Surg 146:1360-1366

12. Smoker WR, Harnsberger HR (1991) Differential diagnosis of head and neck lesions based on their space of origin. 2. The infrahyoid portion of the neck. AJR Am J Roentgenol 157:155-159
13. Kumka M, Bonar J (2012) Fascia: a morphological description and classification system based on a literature review. J Can Chiropr Assoc 56:179-191

14. Standring S (ed) (2004) Gray's anatomy: the anatomical basis of clinical practice. Churchill Livingstone, Oxford

15. Warshafsky D, Goldenberg D, Kanekar SG (2012) Imaging anatomy of deep neck spaces. Otolaryngol Clin N Am 45:1203-1221

16. Macéa JR, Fregnani JHTG (2006) Anatomy of the thoracic wall, axilla and breast. Int J Morphol 24:691-704

17. Zylak CM, Standen JR, Barnes GR, Zylak CJ (2000) Pneumomediastinum revisited. Radiographics 20:1043-1057

18. Skandalakis $\mathrm{PN}$, Zoras $\mathrm{O}$ et al (2006) Transversalis, endoabdominal, endothoracic fascia: who's who? Am Surg 72:16-18

19. Lidid L, Valenzula J, Villarroel C, Alegria J (2013) Crossing the barrier: when the diaphragm is not a limit. AJR Am J Roentgenol 200:W62W70

20. Oliphant M, Berne AS, Meyers MA (1999) The subserous thoracoabdominal continuum: embryologic basis and diagnostic imaging of disease spread. Abdom Imaging 24:211-219

21. Kleinman P, Brill P, Whalen J (1978) Anterior pathway for transdiaphragmatic extension of pneumomediastinum. AJR Am J Roentgenol 131:271-275

22. Lee SL, Ku YM, Rha SE (2010) Comprehensive reviews of the interfascial plane of the retroperitoneum: normal anatomy and pathologic entities. Emerg Radiol 17:3-11

23. Tirkes T, Sandrasegaran K, Patel AA et al (2012) Peritoneal and retroperitoneal anatomy and its relevance for cross-sectional imaging. Radiographics 32:437-451

24. Gore R, Balfe DM, Aizenstein RI, Silverman PM (2000) The great escape: interfascial decompression planes of the retroperitoneum. AJR Am J Roentgenol 175:363-370

25. Love L, Meyers M, Churchill R, Reynes C, Moncada R, Gibson D (1981) Computed tomography of extraperitoneal spaces. AJR Am J Roentgenol 136:781-789

26. Meyers MA (1974) Radiological features of the spread and localization of extraperitoneal gas and their relationship to its source. Radiology 111:17-26

27. Lim JH, Kim B, Auh YH (1998) Anatomical communications of the perirenal space. Br J Radiol 71:450-456

28. Aikawa H, Tanoue S, Okino Y, Tomonari K, Miyake H (1998) Pelvic extension of retroperitoneal fluid: analysis in vivo. AJR Am J Roentgenol 171:671-677

29. Chen N, Min PO, Liu ZY et al (2010) Radiologic and anatomic study of the extraperitoneal space associated with the rectum. AJR Am J Roentgenol 194:642-652

30. Auh YH, Rubenstein WA, Schneider M et al (1986) Extraperitoneal paravesical spaces: CT delineation with US correlation. Radiology 159:319-328

31. Meyers M (2000) Dynamic radiology of the abdomen: normal and pathologic anatomy, 5th edn. Springer, New York

32. Tan CH, Vikram R, Boonsirikamchai P et al (2011) Pathways of extrapelvic spread of pelvic disease: imaging findings. Radiographics 31:117-133

33. Bhosale PR, Patnana M, Viswanathan C, Szklaruk J (2008) The inguinal canal: anatomy and imaging features of common and uncommon masses. Radiographics 28:819-835 